\title{
An Rb1-dependent amplification loop between Ets1 and Zeb1 is evident in thymocyte differentiation and invasive lung adenocarcinoma
}

\author{
Kevin C Dean' ${ }^{1}$ Li Huang ${ }^{1,4}$, Yao Chen ${ }^{1,5}$, Xiaogin Lu ${ }^{1}$ and Yongqing Liu ${ }^{1,2,3^{*}}$
}

\begin{abstract}
Background: Ras pathway mutation leads to induction and Erk phosphorylation and activation of the Ets 1 transcription factor. Ets1 in turn induces cyclin E and cyclin dependent kinase (cdk) 2 to drive cell cycle progression. Ets1 also induces expression of the epithelial-mesenchymal transition (EMT) transcription factor Zeb1, and thereby links Ras mutation to EMT, which is thought to drive tumor invasion. Ras pathway mutations are detected by the Rb1 tumor suppression pathway, and mutation or inactivation of the Rb1 pathway is required for EMT.

Results: We examined linkage between Rb1, Ets1 and Zeb1. We found that an Rb1-E2F complex binds the Ets 1 promoter and constitutively limits Ets1 expression. But, Rb1 repression of Zeb1 provides the major impact of Rb1 on Ets1 expression. We link Rb1 repression of Zeb1 to induction of miR-200 family members, which in turn target Ets1 mRNA. These findings suggest that Ets1 and Zeb1 comprise an amplification loop that is dependent upon miR-200 and regulated by Rb1. Thus, induction of Ets 1 when the Rb1 pathway is lost may contribute to deregulated cell cycle progression through Ets1 induction of cyclin E and cdk2. Consistent with such an amplification loop, we correlate expression of Ets1 and Zeb1 in mouse and human lung adenocarcinoma. In addition we demonstrate that Ets1 expression in thymocytes is also dependent upon Zeb1.
\end{abstract}

Conclusions: Taken together, our results provide evidence of an Rb1-dependent Ets1-Zeb1 amplification loop in thymocyte differentiation and tumor invasion.

Keywords: Ets1, Zeb1, miR-200, Thymocyte differentiation, Lung adenocarcinoma

\section{Background}

c-Ets1 was identified as a proto-oncogene based on v-ets in the genome of the avian leukemia retrovirus E26, and is the founding member of the Ets family of transcription factors [1]. Elevated Ets1 expression has been observed in many invasive and metastatic solid tumors [2] and Overexpression of Ets1 is sufficient for transformation of NIH3T3 fibroblasts [3]. In adults, expression of Ets1 becomes restricted primarily to lymphoid tissues [4], and mutation of Ets1 in mice leads to defects in maturation of lymphocytes [5-7]. Ets1 interacts with Tlx to cause the critical maturation arrest in $\mathrm{T}$ cell acute lymphoblastic

\footnotetext{
* Correspondence: y0liu016@louisville.edu

'Department of Ophthalmology and Visual Sciences, University of Louisville Health Sciences Center, 301 E. Muhammad Ali Blvd., Louisville, KY 40202, USA ${ }^{2} J a m e s$ Graham Brown Cancer Center, University of Louisville Health Sciences Center, Louisville, KY 40202, USA

Full list of author information is available at the end of the article
}

leukemia [8]. Induction of Ets1 in solid tumors triggers neovascularization and the epithelial-mesenchymal transition (EMT) that drives tumor invasion $[9,10]$. Ras pathway signaling is critical for normal development, and constitutively activating Ras mutations in tumors short-circuit the pathway leading to growth factor-independent cell proliferation, neovascularization and EMT [11,12]. Ets1 is phosphorylated and activated by Erk phosphorylation when the Ras pathway is engaged [13-15], and this induction of Ets1 is a mediator of Ras-initiated EMT. Accordingly, a downstream target of Ets1 is the EMT transcription factor Zeb1 [16], which is required for maintaining epithelial vs. mesenchymal balance in vivo [9]. When induced in response to Ras mutation, Zeb1 causes transition to an invasive mesenchymal phenotype [17]. A key sensor of mutant Ras is the Rb1 family of cell cycle regulators, whose activation in response to Ras mutation represses Zeb1 and blocks EMT [18]. 
Recent studies have found that Ets is repressed by miR-200 family members [19]. miR-200 also represses Zeb1, but in a double negative loop Zeb1 binds the promoters of miR-200 family members and represses their expression [20,21]. Such findings raised the possibility that Zeb1 might feedback to induce Ets1 via its repression of miR-200, and that Rb1 might also influence Ets1 expression via its regulation of Zeb1. Here, we examined potential linkage between Rb1, Ets1, and Zeb1. Although Rb1 can interact with genes in a cell cycle-dependent fashion to regulate proliferation, it is also found constitutively at other genes including pro-apoptotic factors and mutation or inactivate of Rb1 is required for induction of such genes [22]. We found here that Rb1 is present constitutively at the Ets1 promoter and removal of an Rb1-E2F complex using a dominant negative-E2F led to induction of Ets1. Thus, Rb1 directly diminishes the level of Ets1 expression. We also provide evidence that Zeb1 induces Ets1, and we show that an additional and major effect of Rb1 on Ets1 expression is mediated through $\mathrm{Rb}$ repression of Zeb1. We link the effect of Zeb1 to its regulation of miR-200, which in turn target Ets1.

Taken together, our results provide evidence of an amplification loop consisting of Ets1 and Zeb1, which is mediated by miR-200 and regulated by Rb1. We also show that Zeb1 and Ets1 are expressed together at the invasive edge of K-Ras-initiated mouse lung adenocarcinomas, and there is a significant correlation between expression of Ets1 and Zeb1 in human lung adenocarcinoma. Like Ets1, Zeb1 is important for thymocyte differentiation, and $Z e b 1(-/-)$ mice show a reduction in thymocytes [5,23-27]. Importantly, we demonstrate that mutation of Zeb1 eliminates Ets1 expression in thymocytes, demonstrating dependence of Ets1 expression on Zeb1 in thymocytes, and thus potentially linking the Zeb1 phenotype in T cell differentiation to a lack of Ets1 expression.

\section{Methods}

\section{Cells and cell culture}

$\mathrm{Rb}$ family triple knockout (TKO) mouse embryo fibroblasts and control wild-type fibroblasts have been described and were a kind gift from T. Jacks and J. Sage [28]. Three independent TKO and wild-type isolates were used with similar results. Mouse Zeb1 wild type and mutant fibroblasts were isolated from crosses of mice heterozygous for Zeb1 and genotyped as described [23]. The human osteosarcoma U2OS cells expressing IPTG-inducible p16 ${ }^{\text {INK4a }}$ were described previously [29], as were the U2OS cells expressing both IPTG-inducible p16 ${ }^{\mathrm{INK} 4 \mathrm{a}}$ and DN-E2F-mER [30]. U2OS cells were cultured with $1 \mathrm{mM}$ IPTG in the medium for either one or three days to induce $\mathrm{p} 16^{\mathrm{INK} 4 \mathrm{a}}$, or with $100 \mathrm{nM}$ Tamoxifen $(\mathrm{OHT})$ for one day to induce mER-DB-E2F expression. For combined treatments, cells were treated with IPTG for one day, and then OHT was added along with the IPTG for an additional day. All the above fibroblast cells were cultured in DMEM medium with $10 \%$ fetal bovine serum (FBS) and antibiotics at $10 \% \mathrm{CO}_{2}$ and $37^{\circ} \mathrm{C}$.

\section{Zeb1 shRNA construct and lentivirus preparation}

We have described the lentiviral shRNA knockdown of Zeb1 protein and mRNA in detail previously $[17,18,31,32,33]$.

\section{Immunohistochemistry}

Mouse embryos and adult lung tumor tissues were fixed with $10 \%$ formalin, paraffin-embedded, and sectioned at $5 \mu \mathrm{m}$. Primary antibodies for Zeb1 (kind gift from Dr. Douglas Darling), CD3 (kind gift from Dr. Qingxian Lu), E-cadherin (Santa Cruz Technology), Ets1 (Santa Cruz Biotechnology) and the secondary antibodies are detailed in Additional file 1: Table S1. The slides were mounted with coverslips using anti-fade medium Permount with DAPI (Fisher) and viewed under a Zeiss fluorescent microscope.

\section{K-Ras ${ }^{\mathrm{LA} 1}$ mice}

Housing and handling of all mice was in accordance with procedures approved by the University of Louisville Institutional Animal Care and Use Committee (IACUC). K-Ras ${ }^{\text {LA1 }}$ mice [34] in a C57BL6 background were obtained from Jackson Laboratory. PCR genotyping was as described previously [34].

\section{Human lung adenocarcinoma microarray analysis}

No human tissue was utilized in this study. The processed microarray data were used from previously published work (the NCBI database GSE_1969163) that contains 59 samples of human lung adenocarcinomas sequenced for mutations in K-RAS and EGFR and patient matched control lung tissue [35]. The expression levels of Zeb1 and Est1 mRNAs were selected for calculating Pearson correlation score.

\section{Chromatin immunoprecipitation (ChIP) assay}

ChIP assays were based on the UpState protocol (http:// www.tc.umn.edu/ muell002/Laboratory/protocols/X-ChIP. htm) using formaldehyde to crosslink genomic DNA of wild-type MEF cells. The chromatin was sheared to an average length of 300-500 bp. Monoclonal antibodies for Rb (Santa Cruz sc-50), E2F1 (Santa Cruz sc-193), E2F4 (Santa Cruz sc-866), and polyclonal antiserum for Zeb1 (a kind gift from Dr. Douglas Darling) were used for immunoprecipitation. Equal amounts of anti-IgG or pre-immune serum were used as controls. The sequence of primers for Ets1 promoter and the expected size of the PCR products are show in Additional file 2: Table S2. ChIP PCR reactions 
were similar to those described below for real-time PCR, but with additional 1\% BSA and 1\% DMSO, and the PCR programs usually had a higher annealing temperature (e.g. $60-68^{\circ} \mathrm{C}$ ) and longer extension time (e.g. 1 minute).

\section{Protein extraction and electrophoretic mobility shift assay (EMSA)}

The cultured adherent wild-type MEF cells were scraped off and lysed in the lysis buffer $(50 \mathrm{mM}$ Tris/ $\mathrm{HCl}, \mathrm{pH} 7.4$, $100 \mathrm{mM} \mathrm{NaCl}, 0.5 \%$ Triton X-100, 0.5\% NP-40) on ice for 20 minutes, followed by 10 -minute centrifuge at 13,000 g, the supernatant was thereafter collected as non-denatured protein extract immediately for the gel shift assay. Based on the sequence alignment of both human and mouse Ets1 promoters, an aligned 24-bp short sequence (5'-TCCATAATTTGCCACTGATAGAT-3') with a putative E2F site (TTTGCCAC) is selected for synthesis of the doublestrand oligos with and without artificial mutation of the E2F site (the mutant oligo sequence: $5^{\prime}$-TCCATAACCTAGCTAGATAGAT-3'). Twenty $\mu \mathrm{g}$ of the above crude protein lysate was mixed with $10 \mathrm{pmol}$ of each of the double-strand oligos in a 10- $\mu$ l protein-DNA binding buffer (5 mM Tris/HCl pH7.4, $100 \mathrm{mM} \mathrm{KCl,} 100 \mu \mathrm{M}$ dithiothrreitol, $100 \mu \mathrm{M}$ EDTA), and incubated at RT for 40 minutes. The binding reactions without protein lysate or oligos were served as background controls. All the binding reaction samples were separated on a $6 \%$ native polyacrylamide gel in $0.5 \times \mathrm{TBE}$ buffer at constant $200 \mathrm{~V}$ and RT for 20 minutes. The finished gel was stained with 10,000 $\times$ diluted SYBR green (Molecular Probe) in $0.5 \times$ TBE buffer at RT for 20 minutes, and then visualized under UV light.

\section{RNA extraction and real-time PCR}

Total cellular RNA was extracted using TRIzol solution (Invitrogen, Carlsbad, California). Using Primer3 (http:// bioinfo.ut.ee/primer3-0.4.0/), primer sets were designed to generate $150-250$ base pair PCR products that bridge two separate exons. Primer sequence, melting temperature $(\mathrm{Tm})$, and PCR product sizes are listed in Additional file 2: Table S2. First-strand complementary DNA (cDNA) synthesis was carried out in 20- $\mu \mathrm{l}$ reactions containing 1-2 $\mu \mathrm{g}$ of total RNA, $500 \mathrm{ng}$ random hexamers, $10 \mathrm{mM}$ dithiothreitol, $500 \mu \mathrm{M}$ dNTP mix, $40 \mathrm{U}^{\mathrm{RNaseOUT}} \mathrm{T}^{\mathrm{mm}}$ ribonuclease inhibitor, and $200 \mathrm{U}$ M-MLV reverse transcriptase, at $37^{\circ} \mathrm{C}$ for $1 \mathrm{~h}$ according to the manufacturer's protocol (Invitrogen, Carlsbad, California). Real-time quantitative PCR was performed in $25-\mu \mathrm{l}$ reaction volumes containing $0.25-\mu \mathrm{l}$ aliquots of cDNA, gene-specific primer pairs, and SYBR Green I fluorescent dye (Molecular Probes, Eugene, Oregon), in an Mx3000P Real-Time PCR System (Stratagene, Cedar Creek, Texas), according to the manufacturer's instructions. The PCR cycle parameters were set at $95^{\circ} \mathrm{C}$ for $20 \mathrm{sec}, 60^{\circ} \mathrm{C}$ for $30 \mathrm{sec}$, and $72^{\circ} \mathrm{C}$ for $30 \mathrm{sec}$, for a total of not more than 45 cycles. The fluorescent intensity of SYBR green was monitored at the end of each extension step; relative amounts of the target cDNA was estimated by the threshold cycle $(\mathrm{Ct})$ number, and normalized to two internal control genes, $\beta$-actin (ACTB) and glyceraldehyde-3phosphate dehydrogenase (GAPDH). Three independent samples were analyzed for each condition and/or cell type, and each sample was compared in at least 3 independent RT-PCR amplifications.

\section{MicroRNA detection}

The quantitative RT-PCR to detect miRNAs is as described [36]. Briefly, polyadenylation of at least $5 \mu \mathrm{g}$ of the total RNA was completed by poly(A) polymerase kit (PAP, Ambion) in a $20 \mu \mathrm{l}$ of reaction volume according to manufacturer's instruction. The polyadenylated RNA was thereafter directly utilized for cDNA preparation using a reverse transcription kit (M-MLV reverse transcriptase, Invitrogen) and an adaptor primer (5'-GCGAGCACAGAATTAATACGACTCACTATAGG(T) ${ }_{12} \mathrm{VN}^{*}-3^{\prime}$ ) in a $40 \mu \mathrm{l}$ of reaction volume. Real-time quantitative PCR was performed using a universal primer (5'-GCGAGCACAGAATTAATACGAC-3') and a miRNA-specific primer (Additional file 2: Table S2) as described above.

\section{Results}

\section{The $\mathrm{Rb} 1$ family represses Ets 1 expression}

Mutations in Ras trigger activation of $\mathrm{Rb} 1$ and tumor suppressing oncogene-induced senescence $[17,37]$. Thus, mutation or inactivation of the Rb1 pathway, which is a hallmark of cancers, is critical for Ras-initiated tumor formation. Ets1 is phosphorylated and activated by Erk in response to Ras signaling [13], and in turn Ets1 induces cyclin $\mathrm{E}$ and cdk2 to drive cell cycle progression [38]. Therefore, we wondered if Rb1 might repress Ets1. Indeed, mutation of $\mathrm{Rb} 1$ in primary cultures of mouse embryo fibroblasts (MEFs) led to induction of Ets1, and Ets1 was further induced when all three closely related Rb1 family members (Rb1, Rbl1 and Rbl2) were mutated to generate triple knockout (TKO) MEFs (Figure 1). These results suggest that Ets1 is under repression by the Rb1 family.

\section{E2F1-Rb1 binds the Ets1 promoter and constitutively repressed the gene}

We used chromatin immunoprecipitation (ChIP) assay and electrophoretic mobility shift assay (EMSA) to determine whether Rb1 is bound to the Ets1 promoter and whether removal of its putative binding site of E2F family, the Rb1 DNA binding partner, diminishes the binding. Rb1 does not bind directly to DNA, but it can be tethered to promoters through interaction with the E2F family of transcription factors [39]. Some E2Fs (e.g. E2F1) contain a transactivation domain, while other E2Fs lack a transactivation domain (e.g., E2F4). Binding to Rb1 blocks the transactivation domain of E2Fs, but $\mathrm{Rb} 1$ also interacts 


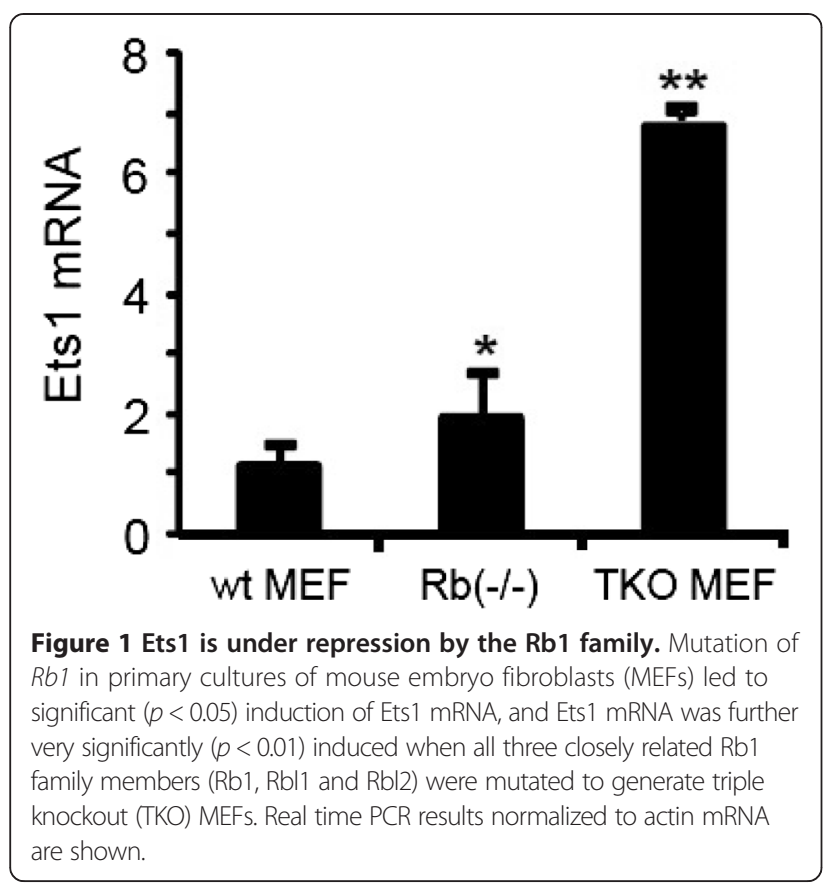

Figure 1 Ets 1 is under repression by the Rb1 family. Mutation of $R b 1$ in primary cultures of mouse embryo fibroblasts (MEFs) led to significant $(p<0.05)$ induction of Ets 1 mRNA, and Ets1 mRNA was further very significantly $(p<0.01)$ induced when all three closely related Rb1 knockout (
are shown. with epigenetic modifiers to form an active repressor [29]. Both human and mouse Ets1 promoters have two major similar regions that each contains a perfectly aligned consensus E2F binding site (Figure 2A and B). ChIP assays showed that Rb1 and E2F1, but not E2F4, were bound to the Ets1 promoter in vivo (Figure 2C), while EMSA demonstrated that mutation of the putative E2F site reduced the specific binding of the crude protein lysate to the target DNA oligo in vitro (Figure 2D), suggesting that Rb1E2F1 complex specifically binds to the putative E2F site of the Ets1 promoter. Next, we wondered if Rb1 was acting at the Ets1 promoter to block transactivation by E2F, or if $\mathrm{Rb} 1$ was serving as an active repressor. Previously we described a dominant negative (DN) form of E2F consisting of the DNA binding domain fused to a mutant estrogen receptor (DN-E2F-mER). Following hydroxyl-tamoxifen $(\mathrm{OHT})$ treatment, DN-E2F-mER is translocated to the nucleus where it displaces E2F and E2F-Rb1 complexes from promoters. In the same cell, an IPTG-inducible $p 16$ gene is also included to assemble E2F-Rb complexes at target genes following IPTG treatment [40] (Figure 2A). If E2F were serving a transactivation function at the Ets1 promoter, then DN-E2F-mER would cause repression, but if an E2F-Rb1 complex were serving as a constitutive repressor, DN-E2F-mER would cause induction. We found that

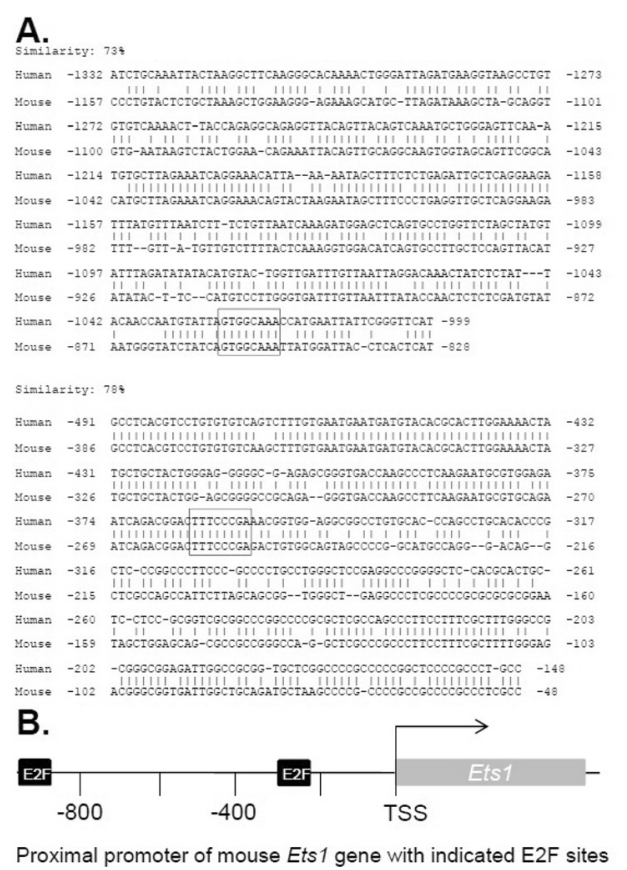

C.

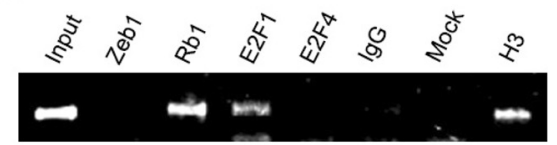

D.

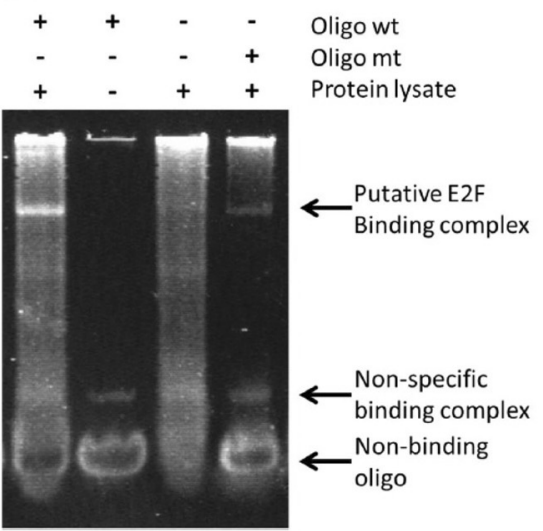

Figure 2 An Rb1-E2F1 complex binds the Ets 1 promoter and constitutively represses the gene. (A) Alignment of both human and mouse Ets 1 promoter sequences showing two regions of higher similarity that contains consensus E2F binding sites (boxed). (B) A schematic diagram of mouse Ets 1 promoter with two putative E2F sites. SST: start site of transcription. (C) Chromatin immunoprecipitation (ChIP) assay demonstrates binding of Rb1 and E2F1 to the Ets 1 promoter together with the positive control histone H3. Input indicates the DNA used for immunoprecipitation, IgG is control serum and mock indicates no added DNA. (D) Electrophoretic mobility shift assay (EMSA) demonstrates reduction of the specific binding of the crude protein lysate to the target DNA oligo upon mutation of its putative E2F site. 'wt' denotes a wild-type oligo with a putative E2F site while ' $m t^{\prime}$ indicates a mutant oligo with a mutated E2F site. 
A.

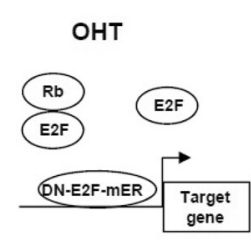

D. 3.5

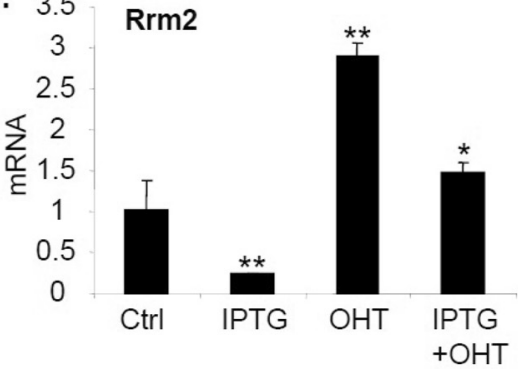

B.
C. 6
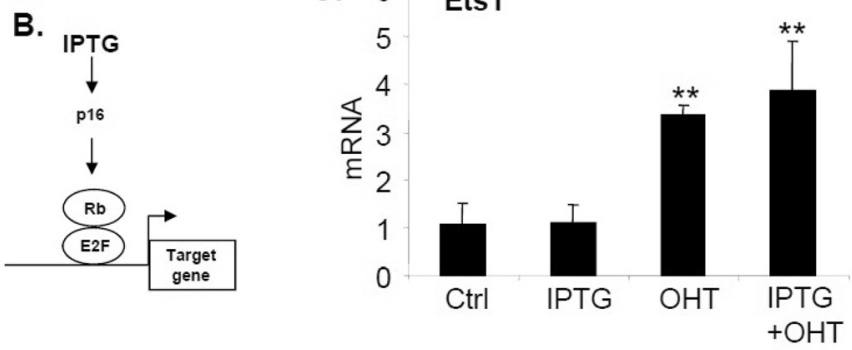

E. $25 \mathrm{Ntf3}$

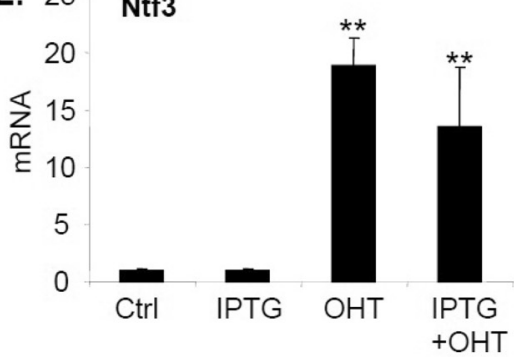

Figure 3 Regulation of Ets1, Rrm2 and Ntf3 expression by Rb-E2F complex. (A) Diagram showing a dominant negative (DN) form of E2F where the E2F DNA binding domain is fused to the mutant hydroxyl-tamoxifen (OHT)-dependent estrogen receptor ( $\mathrm{m}$-ER) displacing E2F and $\mathrm{Rb}-\mathrm{E} 2 \mathrm{~F}$ from a promoter following OHT treatment. IPTG-inducible p16 expression is shown leading to assembly of E2F-Rb complexes at target genes [40]. (B) Human U2OS cells were treated with IPTG, OHT or IPTG + OHT as described in Material and Methods. Real time PCR was used to measure mRNA levels of Ets1 (C), Rrm2 (D) and Ntf3 (E). "Ctrl" indicates untreated control cells. Results were normalized to $\beta$-actin mRNA. Rrm2, ribonucleotide reductase, Ntf3, neurotrophin 3.
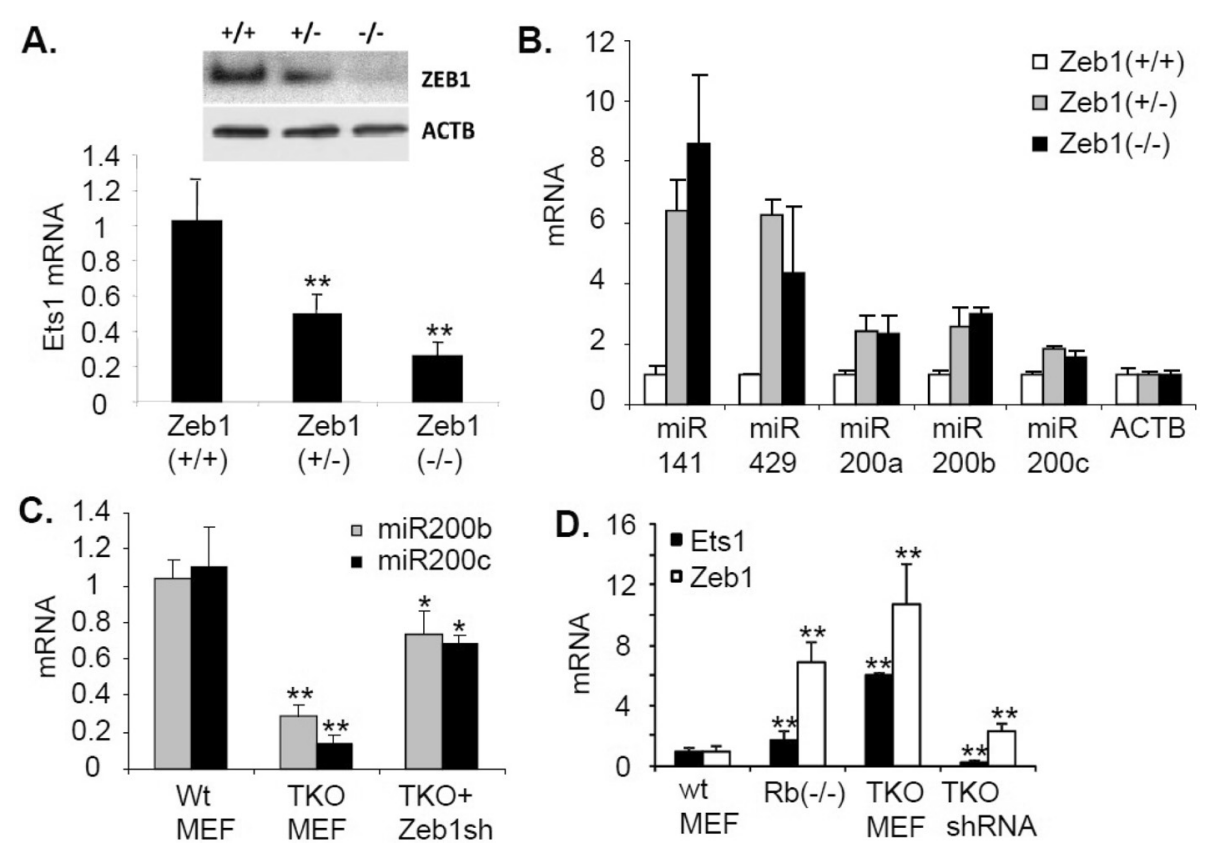

Figure 4 Zeb1 induces Ets1 expression, and it mediates much of the effect of Rb1 on Ets1 expression. (A) Zeb1 mutation leads to a gene dosage-dependent downregulation of Ets1 expression. Real time PCR is shown and results are normalizaed to $\beta$-actin mRNA when the Western blots show similar result accordingly. (B) Zeb1 mutation leads to a gene dosage-dependent induction of miR-200 family members. Real time PCR is shown. (C) Rb1 family mutation leads to induction of miR-200 family members, and this induction is reversed by lentiviral knockdown of Zeb1 (Zeb1 sh). (D) Zeb1 is induced along with Ets1 as Rb1 family members are mutated. And, knockdown of Zeb1 reverses most of the induction of Ets1. Real time PCR is shown. 


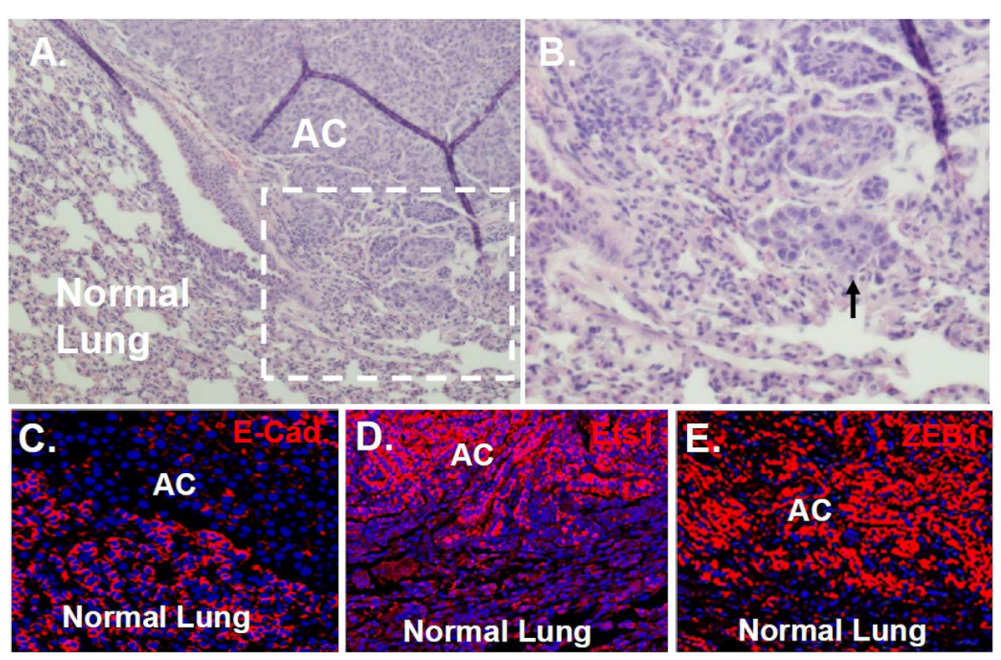

Figure 5 Ets1 and Zeb1 are expressed at the invasive front of K-Ras initiated mouse lung adenocarcinoma. (A and B) H\&E staining showing the invasive front of an adenocarcinoma (AC) from a K-Ras ${ }^{\text {LA1 }}$ mouse lung. (C-E) Note immunostaining for E-cadherin (E-cad.) in normal lung and expression of Zeb1 and Est1 in carcinoma invading into normal lung. Arrows indicate invasive carcinoma.

following tamoxifen treatment, DN-E2F-mER caused induction of Ets1, demonstrating that E2F1-Rb1 at the promoter is constitutively repressing Ets1 (Figure 3C). Rb1 activity is regulated in a cell cycle dependent fashion by cyclin dependent kinases (cdks) that hyperphosphorylate the protein blocking its interaction with E2F [22]. Cdk inhibitors such as p16 block the activity of these cdks and cause accumulation of constitutively active Rb1 which associates with E2F at the promoter of target genes [41]. Many genes such as Rrm2 are dependent upon phosphorylation/dephosphorylation of Rb1 during the cell cycle and these genes are further repressed when Rb1 is activated by expression of p16 (Figure 3D), but other genes such as Ntf3 that are constitutively repressed by Rb1-E2F are not further repressed by activation of $\mathrm{Rb} 1$ through expression of p16 (Figure 3E). We previously described expression of p16 driven by an IPTG-inducible promoter [32] (Figure 3B). As with Ntf3, we found that induction of p16 had no effect on repression of Ets1 (Figure 3C). Taken together, these results suggest that E2F-Rb1 is present constitutively at the Ets1 promoter, where it reduces the level of Ets1 expression in a cell cycle-independent fashion.

\section{Zeb1 repression of the miR-200 family is linked to induction of Ets 1}

Zeb1 is a target of the miR-200 family, but in a negative loop Zeb1 feeds back to repress transcription of miR-200 family members [20,21]. Because miR-200 has been shown to target Ets1 [19], we wondered if Zeb1 might also affect expression of Ets1. Indeed, we found that mutation of Zeb1 in MEFs led to a gene dosage-dependent increase in expression of Ets1 (Figure 4A). We did not detect Zeb1 at the Ets1 promoter in ChIP assays (Figure 2C) and induction of Ets1 coincided with induction of miR-200 as Zeb1 alleles were mutated (Figure 4B), suggesting that it is induction of miR-200 when Zeb1 is mutated that is causing induction of Ets1. It is of note that further downregulation of Zeb1 in Zeb1 null MEF cells had little effects on miR-200 expression (Figure 4B), possibly due to [1] the level of Zeb1 protein in het MEF cells is low enough to relieve the repression on miR-200 family, further decrease in Zeb1 expression in null cells will not increase in miR-200 expression accordingly or/and [2] it is also possible that Zeb1 might down-regulate other unknown factor(s) that positively regulates expression of miR-200 family at the same time and thereby makes the regulation network more complex. Taken together, these results suggest an

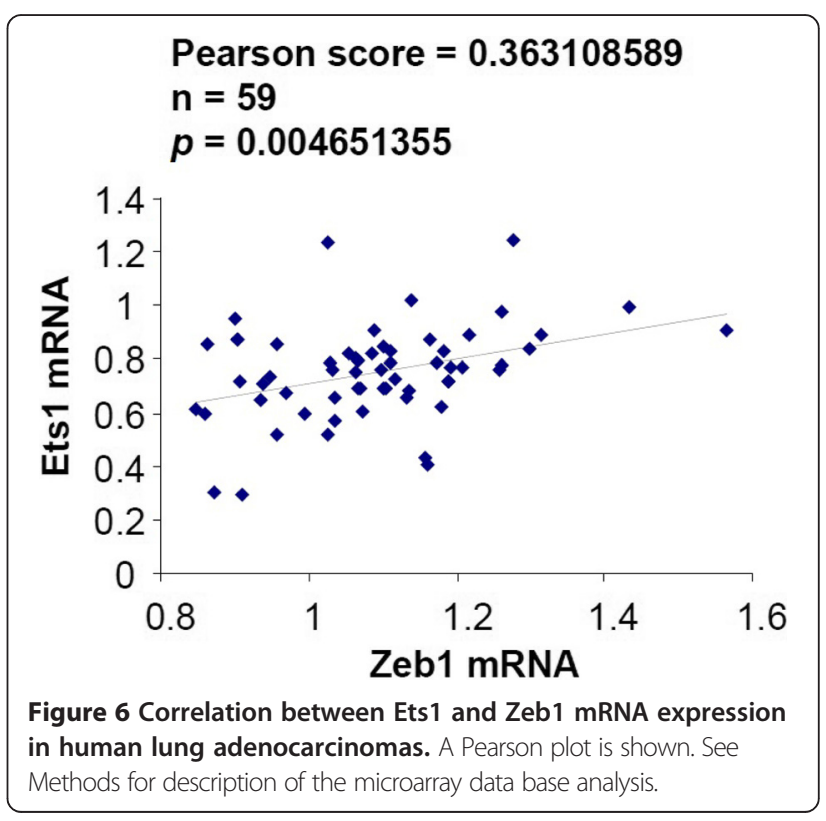


amplification loop between Ets1 and Zeb1, where Ets1 increases transcription of Zeb1, and Zeb1 in turn represses miR-200 to further elevate Ets1.

\section{$\mathrm{Rb} 1$ family repression of Zeb1 is a major component of inhibition of Ets1 by Rb1}

Even though an Rb1-E2F complex binds to the Ets1 promoter to reduce its expression, it is of note that $\mathrm{Rb} 1$ also represses Zeb1 [40], leading us to ask whether Rb1 repression of Zeb1 was also contributing to the Rb1dependent downregulation of Ets1. As we demonstrated previously [16], mutation of Rb1 family members led to induction of Zeb1 and this induction of Zeb1 was accompanied by repression of miR-200 (Figure 4C) and, as shown above, Ets1 (Figure 1). Knockdown of Zeb1 in the TKO MEFs using shRNA lenvirirus, as described previously [17], eliminated much of the induction of Ets1 (Figure 4D), suggesting that induction of Zeb1 and repression of miR-200 as $R b 1$ family members are mutated is contributing significantly to the upregulation of Ets1. These results suggest that Rb1-E2F binds the Ets1 promoter to limit its level of expression, but it is induction of Zeb1 and in turn repression of miR-200 when the Rb1 family activity is lost that is responsible for most of the induction of Ets1.

\section{Ets1 and Zeb1 are expressed at the invasive front} of K-Ras initiated mouse lung adenocarcinoma

Ras pathway signaling causes Erk-dependent activating phosphorylation of Ets1 [13] and the resulting induction of Zeb1 is thought to be an important factor in Rasinitiated EMT (which is opposed by Rb1) [9]. Induction of Zeb1 leads to transition to a migratory mesenchymal phenotype and can been seen at the invading front of tumors [17]. We examine expression of Ets1 and Zeb1 in $\mathrm{K}$-Ras initiated mouse lung adenocarcinomas (Figure 5A and B). Activation of an oncogenic K-Ras allele by recombination in somatic cells is sufficient for spontaneous lung cancer in K-Ras ${ }^{\mathrm{LA} 1}$ mice [34]. In this mouse model regions of atypical adenomatous hyperplasia (AAH) becomes evident in bronchial airways, and this hyperplasia expands to initiate formation of subpleural precancerous adenomas. Foci of adenocarcinoma in situ begin to appear within these precancerous lesions as the first evidence of transition to malignancy, and these adenocarcinomas subsequently expand and invade into surrounding lung tissue where E-cadherin is expressed (Figure 5C). In this model, Rb1 is a major barrier to tumor initiation, and it is inactivated by cdk hyperphosphorylation in these tumors [28]. Neither Ets1 nor Zeb1 were expressed in precancerous AAH or adenomas, but both were expressed at the invasive edge of adenocarcinomas (results not shown; Figure 5D and $\mathrm{E})$.

\section{Correlation between Ets1 and Zeb1 in human lung adenocarcinomas}

To check if Ets1 also follows Zeb1 expression in human lung adenocarcinomas, we examined microarrays of 59 human lung adenocarcinomas [35] and compared expression of Ets1 and Zeb1 mRNAs. We found a significant correlation between expression of Ets1 and Zeb1 mRNA in these tumors (Figure 6). Taken together, these data with mouse and human lung adenocarcinomas are consistent with the amplification loop we described above for Ets1 and Zeb1.

\section{Mutation of Zeb1 eliminates Ets1 expression in differentiating thymocytes}

Although the correlation between Ets1 and Zeb1 in lung adenocarcinoma seen above is consistent with an amplification loop between Ets1 and Zeb1, these results are also consistent simply with the previously documented induction of Zeb1 by Ets1 [9]. Therefore, we next asked whether Ets1 expression is dependent upon Zeb1. Mutation of Zeb1 and Ets1 both lead to defects in thymocyte differentiation [23-27]. Zeb1(-/-) mice show a decrease in the number of thymocytes (Figure 7A, B, C, F, G and H), and we found that this Zeb1 mutation eliminated Ets1 expression in the thymocytes (Figure 7D, E, I and J). These results demonstrate that Ets1 expression in thymocytes is dependent upon Zeb1, and further confirm the linkage between the two transcription factors in the defects leading to thymocyte differentiation.

\section{Discussion}

Mutation in the Ras pathway leading to growth factor independent signaling is frequent in tumors $[11,12]$. One consequence of Ras mutation is EMT, and this appears to be mediated at least in part by Erk phosphorylation and activation of Ets1 and in turn Ets1 induction of the EMT transcription factor Zeb1 [13]. The Ras pathway is under surveillance by the Rb1 tumor suppressor family, and mutation or inactivation of the Rb1 family is a hallmark of cancer required for progression of tumors with a Ras pathway mutation [22]. One consequence of Rb1 activation in response to Ras mutation is to suppress EMT. Our results suggest that E2F-Rb1 binds constitutively to the Ets1 promoter and limits the level of expression, but a second major impact of Rb1 on Ets1 expression is mediated through $\mathrm{Rb} 1$ repression of Zeb1 [40] and in turn induction of miR-200, which targets Ets1. We propose that Ets1 and Zeb1 form an amplification loop that is dependent upon Zeb1 repression of miR-200 and regulated by the Rb1 family (Figure 8). We present evidence correlating expression of Ets1 and Zeb1 in invasive lung adenocarcinoma, suggesting that this amplification loop is evident in invasive lung cancer. Importantly, we demonstrate that Zeb1 is required for Ets1 expression in differentiating thymocytes providing evidence 

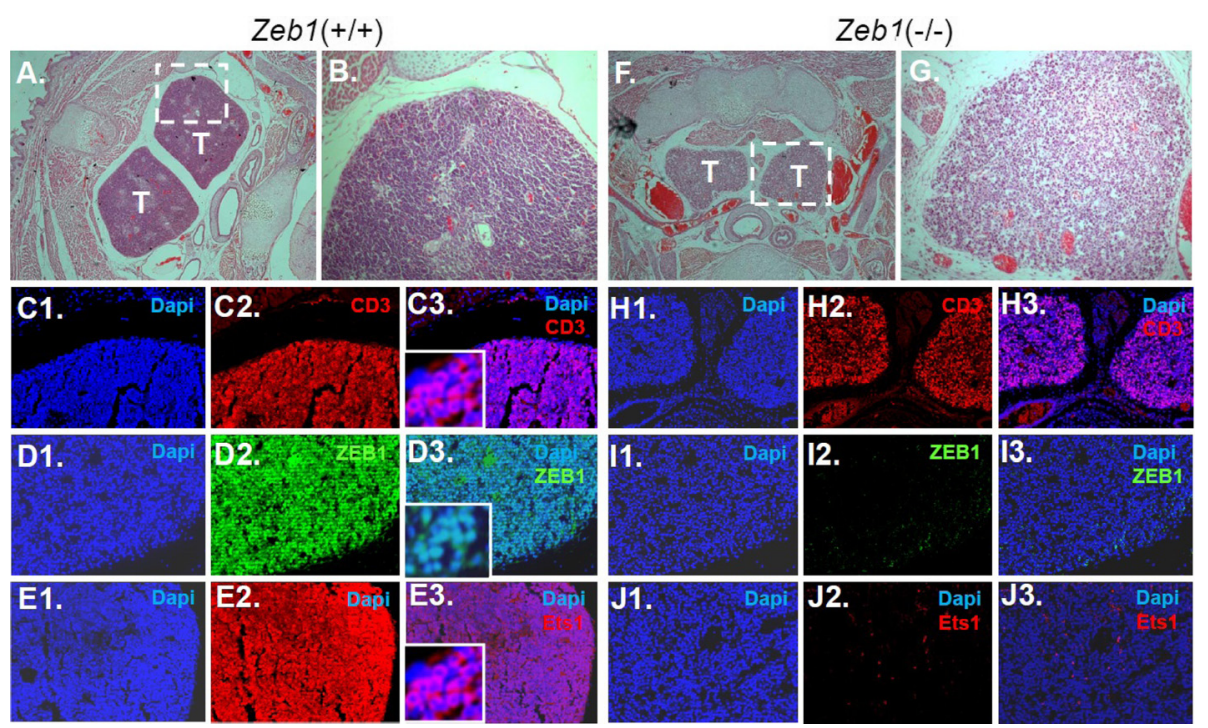

Figure 7 Zeb1 mutation eliminates Ets1 expression in differentiating embryonic thymocytes. A-G H\&E sections of embryonic day 15.5 wild-type and Zeb1(-/-) embryos showing the thymus (T). Note the decrease in thymocyte number with Zeb1 mutation. (C-J) Immunostaining showing loss of expression of Ets1 with Zeb1 mutation in differentiating thymocytes. CD3 is a T-cell marker.

that Zeb1 is critical for Ets1 expression in vivo in the thymus. Beyond regulating expression of Zeb1 and EMT, Ets1 also induces expression of cyclin $\mathrm{E}$ and $\mathrm{cdk} 2$ to drive cell cycle progression [38]. Rb1 pathway mutation or inactivation is closely linked to unrestricted cell proliferation [22], and our results suggest that one component of this unrestricted cell cycle progression might be upregulation of Zeb1 and in turn Ets1 when the Rb1 pathway is lost. Interestingly, two separate transcriptional functions have been ascribed to Zeb1. Zeb1 binds to DNA through a series of zinc finger domains, and a C-terminal domain binds to the co-repressor CtBP to repress transcription [42]. The $\mathrm{N}$-terminal domain of Zeb1 binds Smads and the transcriptional co-activator p300, and Zeb1 acts as a scaffold for assembly of an active smad-p300 complex at promoters of TGF and BMP-responsive genes [43]. Two different

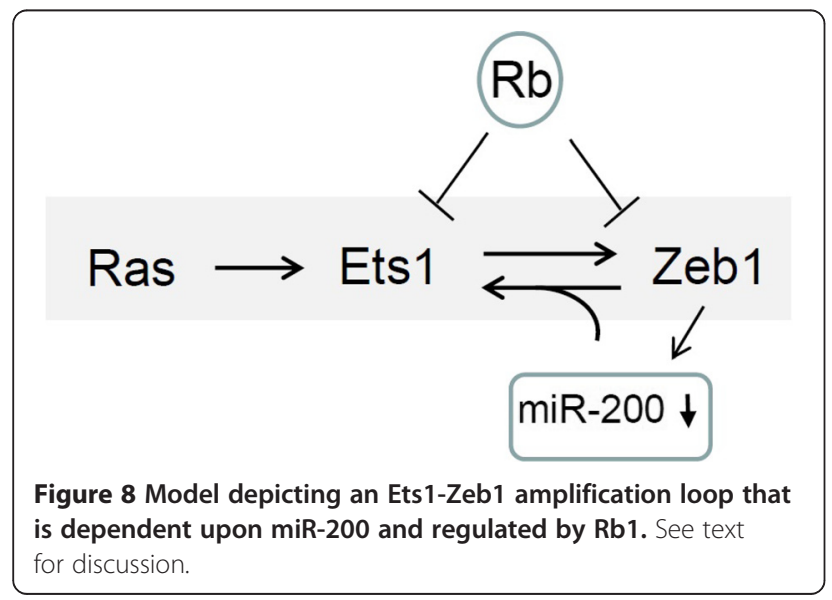

mutations of Zeb1 have been examined-a gene knockout and a mutation that deletes the N-terminal smad-p300 binding domain $[23,30]$. Knockout of Zeb1 leads to lethality at the end of gestation, with cleft palate, skeletal defects, disrupted epithelial vs. mesenchymal balance and diminished thymocyte differentiation [23]. Interestingly, deletion of the $\mathrm{N}$ - terminal domain of Zeb1 eliminates cleft palate and other skeletal defects, but the thymocyte differentiation defect persists [30]. These results imply that CtBPdependent transcription repression of miR-200 family members by Zeb1 is important for regulation of Ets1 and for thymocyte differentiation.

\section{Conclusions}

Our results provide evidence of an Rb1-dependent Ets1-Zeb1 amplification loop that is important in both cancer and in normal development of the thymus.

\section{Additional files}

Additional file 1: Ets1 paper supplementary table 1, $7 \mathrm{~K} \mathrm{http://www.}$ biomedcentral.com/imedia/8917713331383367/supp1.pdf.

Additional file 2: Ets1 paper supplementary table 2, $10 \mathrm{~K}$ http://www. biomedcentral.com/imedia/7163581351383367/supp2.pdf.

\section{Competing interests}

The authors declare that they have no competing interests.

\section{Authors' contributions}

KCD performed most of the experiments and participated in drafting the manuscript. LH performed real time PCR for microRNAs. YC performed EMSA experiments. XL cultured cells and performed immunostaining experiments. $Y L$ planned experiments and wrote the manuscript. All authors read and approved the final manuscript. 


\section{Acknowledgement}

We thank Dr T. Jacks and Dr J. Sage for the gift of TKO and wild-type littermate control MEFs, Dr G. Leone for Rb heterozygous and null cells, Dr. D. Darling for Zeb1 antiserum, and Dr. Qingxian Lu for CD3 antibody. The studies were supported by an Institutional Development Award (IDeA) from the National Institute of General Medical Sciences (P20GM103453) to Y.L.

\section{Author details}

${ }^{1}$ Department of Ophthalmology and Visual Sciences, University of Louisville Health Sciences Center, 301 E. Muhammad Ali Blvd., Louisville, KY 40202, USA. ${ }^{2}$ James Graham Brown Cancer Center, University of Louisville Health Sciences Center, Louisville, KY 40202, USA. ${ }^{3}$ Birth Defects Center, University of Louisville Health Sciences Center, Louisville, KY 40202, USA. ${ }^{4}$ College of Agriculture and Biotechnology, Zhejiang University, Hangzhou, Zhejiang Province 310058 , China. ${ }^{5}$ The Second Affiliated Hospital, Central South University Xiangya School of Medicine, Changsha, Hunan Province 410011, China.

\section{Received: 1 August 2014 Accepted: 26 February 2015} Published online: 19 March 2015

\section{References}

1. Gutierrez-Hartmann A, Duval DL, Bradford AP. ETS transcription factors in endocrine systems. Trends Endocrinol Metab. 2007;18:150-8.

2. Seth A, Watson DK. ETS transcription factors and their emerging roles in human cancer. Eur J Cancer. 2005;41:2462-78.

3. So EN, Crowe DL. Characterization of a retinoic acid responsive element in the human ets-1 promoter. IUBMB Life. 2000;50:365-70.

4. Ghysdael J, Gegonne A, Pognonec P, Dernis D, Leprince D, Stehelin D. Identification and preferential expression in thymic and bursal lymphocytes of a c-ets oncogene-encoded Mr 54,000 cytoplasmic protein. Proc Natl Acad Sci U S A. 1986;83:1714-8.

5. Anderson MK, Hernandez-Hoyos G, Diamond RA, Rothenberg EV. Precise developmental regulation of Ets family transcription factors during specification and commitment to the T cell lineage. Development. 1999;126:3131-48.

6. Barton K, Muthusamy N, Fischer C, Ting CN, Walunas, TL, Lanier $\mathrm{LL}$, et al. The Ets-1 transcription factor is required for the development of natural killer cells in mice. Immunity 1998;9:555-563.

7. Clausen PA, Athanasiou M, Chen Z, Dunn KJ, Zhang Q, Lautenberger JA ETS-1 induces increased expression of erythroid markers in the pluripotent erythroleukemic cell lines K562 and HEL. Leukemia. 1997;1997(11):1224-33.

8. Dadi S, Le Noir S, Payet-Bornet D, Lhermitte L, Zacarias-Cabeza J, Bergeron J, et al. TLX homeodomain oncogenes mediate $T$ cell maturation arrest in T-ALL via interaction with ETS1 and suppression of TCRa gene expression. Cancer Cell. 2012;17:563-76.

9. Shirakihara T, Saitoh M, Miyazono K. Differential regulation of epithelial and mesenchymal markers by deltaEF1 proteins in epithelial mesenchymal transition induced by TGF-beta. Mol Biol Cell. 2007;18:3533-44.

10. Okano K, Hibi A, Miyaoka T, Inoue T, Sugimoto H, Tsuchiya K, et al. Inhibitory effects of the transcription factor Ets-1 on the expression of type I collagen in TGF-ß1-stimulated renal epithelial cells. Mol Cell Biochem. 2012;369:247-54

11. Crowder C, Kopantzev E, Williams K, Lengel C, Miki T, Rudikoff S. An unusual $\mathrm{H}$-Ras mutant isolated from a human multiple myeloma line leads to transformation and factor-independent cell growth. Oncogene. 2003:22:649-59.

12. Liu Y, Dean DC. Tumor initiation via loss of cell contact inhibition versus Ras mutation: do all roads lead to EMT? Cell Cycle. 2010;9:897-900.

13. Chang F, Steelman LS, Lee JT, Shelton JG, Navolanic PM, Blalock WL, et al. Signal transduction mediated by the Ras/Raf/MEK/ERK pathway from cytokine receptors to transcription factors: potential targeting for therapeutic intervention. Leukemia. 2003;17:1263-93.

14. Röttinger $E$, Besnardeau L, Lepage T. A Raf/MEK/ERK signaling pathway is required for development of the sea urchin embryo micromere lineage through phosphorylation of the transcription factor Ets. Development. 2004;131:1075-87.

15. Nelson ML, Kang HS, Lee GM, Blaszczak AG, Lau DK, McIntosh LP, et al. Ras signaling requires dynamic properties of Ets1 for phosphorylation-enhanced binding to coactivator CBP. Proc Natl Acad Sci U S A. 2010;107:10026-31.
16. Dave N, Guaita-Esteruelas S, Gutarra S, Frias À, Beltran M, Peiró S, et al. Functional cooperation between Snail1 and twist in the regulation of ZEB1 expression during epithelial to mesenchymal transition. J Biol Chem. 2011:286:12024-32.

17. Liu Y, Sánchez-Tilló E, Lu X, Huang L, Clem B, Telang S, et al. Sequential inductions of the ZEB1 transcription factor caused by mutation of Rb and then Ras proteins Are required for tumor initiation and progression. J Biol Chem. 2013;288:11572-80.

18. El-Naggar S, Liu Y, Dean DC. Mutation of the Rb1 pathway leads to overexpression of mTor, constitutive phosphorylation of Akt on serine 473, resistance to anoikis, and a block in c-Raf activation. Mol Cell Biol. 2009:29:5710-7.

19. Gill JG, Langer EM, Lindsley RC, Cai M, Murphy TL, Murphy KM. Snail promotes the cell-autonomous generation of Flk1(+) endothelial cells through the repression of the microRNA-200 family. Stem Cells Dev. 2012;21:167-76.

20. Burk U, Schubert J, Wellner U, Schmalhofer O, Vincan E, Spaderna S, et al. A reciprocal repression between ZEB1 and members of the miR-200 family promotes EMT and invasion in cancer cells. EMBO Rep. 2008:9:582-9.

21. Chan, YC, Khanna S, Roy S, and Sen CK. miR-200b targets Ets-1 and is downregulated by hypoxia to induce angiogenic response of endothelial cells. J Biol Chem. 2011,286:2047-56

22. Harbour JW, Dean DC. The Rb/E2F pathway: expanding roles and emerging paradigms. Genes Dev. 2000;14:2393-409.

23. Takagi T, Moribe H, Kondoh H, Higashi Y. DeltaEF1, a zinc finger and homeodomain transcription factor, is required for skeleton patterning in multiple lineages. Development. 1998;125:21-31.

24. Liu Y, El-Naggar S, Darling DS, Higashi Y, Dean DC. Zeb1 links epithelial-mesenchymal transition and cellular senescence. Development 2008;135:579-88

25. Laurent-Huck FM, Egles C, Kienlen P, Stoeckel ME, Felix JM. Expression of the c-ets1 gene in the hypothalamus and pituitary during rat development. Brain Res Dev Brain Res. 1996;97:107-17.

26. Maroulakou IG, Papas TS, Green JE. Differential expression of ets-1 and ets-2 proto-oncogenes during murine embryogenesis. Oncogene. 1994;9:1551-65.

27. Raouf A, Seth A. Ets transcription factors and targets in osteogenesis. Oncogene. 2000;19:6455-63.

28. Sage J, Mulligan GJ, Attardi LD, Miller A, Chen S, Williams B, et al. Targeted disruption of the three $\mathrm{Rb}$-related genes leads to loss of $\mathrm{G}(1)$ control and immortalization. Genes Dev. 2000;14:3037-50.

29. Zhang HS, Postigo AP, Dean DC. Active transcriptional repression by the Rb-E2F complex mediates G1 arrest triggered by p16INK4a, TGF-ß, and contact inh ibition. Cell. 1999:97:53-61.

30. Higashi Y, Moribe H, Takagi T, Sekido R, Kawakami K, Kikutani H, et al. Impairment of $\mathrm{T}$ cell development in deltaEF1 mutant mice. J Exp Med. 1997;185:1467-79.

31. Nishimura G, Manabe I, Tsushima K, Fujiu K, Oishi Y, Imai Y, et al. Delta EF1 regulates TGF-beta signaling in vascular smooth muscle cell differentiation. Dev Cell. 2006;1:93-104.

32. Liu Y, Ye F, Li Q, Tamiya S, Darling DS, Kaplan HJ, et al. Zeb1 represses Mitf and regulates pigment synthesis, cell proliferation, and epithelial morphology. Invest Ophthalmol Vis Sci. 2009;50:5080-8.

33. Tiscornia G, Singer $\mathrm{O}$, Verma IM. Design and cloning of lentiviral vectors expressing small interfering RNAs. Nat Protoc. 2006;1:234-40.

34. Johnson L, Mercer K, Greenbaum D, Bronson RT, Crowley D, Tuveson DA et al. Somatic activation of the K-ras oncogene causes early onset lung cancer in mice. Nature. 2001;410:1111-6.

35. Takeuchi T, Tomida S, Yatabe Y, Kosaka T, Osada H, Yanagisawa K, et al. Expression profile-defined classification of lung adenocarcinoma shows close relationship with underlying major genetic changes and clinicopathologic behaviors. J Clin Oncol. 2006;24:1679-85.

36. Shi $R$, Chiang VL. Facile means for quantifying microRNA expression by real-time PCR. Biotechniques. 2005;3:519-25.

37. Carrière C, Gore AJ, Norris AM, Gunn JR, Young AL, Longnecker DS, et al. Deletion of Rb accelerates pancreatic carcinogenesis by oncogenic Kras and impairs senescence in premalignant lesions. Gastroenterology. 2011;141:1091-101.

38. Sing AK, Swarnalatha M, and Kumar V. c-ETS1 facilitates G1/S-phase transition by up-regulating cyclin E and CDK2 genes and cooperates with hepatitis $B$ virus $X$ protein for their deregulation. J Biol Chem. 2011;286:21961-70 
39. Roland BD, Bernards R. Re-evaluating cell cycle regulation by E2Fs. Cell. 2006;127:1-4.

40. Liu Y, Costantino ME, Montoya-Durango D, Higashi Y, Darling DS, Dean DC. The zinc finger transcription factor ZFHX1A is linked to cell proliferation by Rb-E2F1. Biochem J. 2007;408:79-85.

41. Lowe SW, Sherr CJ. Tumor suppression by INK4a-Arf: progress and puzzles. Curr Opin Genet Dev. 2003;13:77-83.

42. Young AP, Nagarajan R, Longmore GD. Mechanism of transcriptional regulation by $\mathrm{Rb}-\mathrm{E} 2 \mathrm{~F}$ segregates by biological pathway. Oncogene. 2003;22:7209-17.

43. Postigo AA, Dean DC. ZEB represses transcription through interaction with the corepressor CtBP. Proc Natl Acad Sci U S A. 1999;96:6683-8.

\section{Submit your next manuscript to BioMed Central} and take full advantage of:

- Convenient online submission

- Thorough peer review

- No space constraints or color figure charges

- Immediate publication on acceptance

- Inclusion in PubMed, CAS, Scopus and Google Scholar

- Research which is freely available for redistribution 